\title{
High diagnostic accuracy of NT-proBNP for cardiac origin of pleural effusions
}

\author{
M. Kolditz*, M. Halank*, C.S. Schiemanck*, A. Schmeisser ${ }^{\#}$ and G. Höffken*
}

ABSTRACT: A prospective study was performed to evaluate the diagnostic accuracy of $\mathrm{N}$ terminal-pro-B-type natriuretic peptide (NT-proBNP) levels, measured simultaneously in serum and pleural fluid, in identifying pleural effusions due to heart failure.

Pleural fluid and serum samples from all patients presenting for thoracentesis between April 2004 and May 2005 were simultaneously collected. The discriminative properties of NT-proBNP levels in identifying pleural effusions due to heart failure were determined by receiver operating characteristic curve analysis and compared to the diagnostic value of finding a transudate by Light's criteria.

Ninety-three patients were evaluated, $27 \%$ with cardiac effusion and $73 \%$ with exudative effusions of various cause. Levels of NT-proBNP in pleural fluid and serum correlated closely. Serum and pleural fluid NT-proBNP levels were significantly elevated in patients with cardiac effusion. With a cut-off value of $4,000 \mathrm{ng} \cdot \mathrm{L}^{-1}$, NT-proBNP levels in pleural fluid and serum displayed comparably high diagnostic accuracies of 92 and $91 \%$, respectively. All patients misclassified by Light's criteria were correctly identified by measuring NT-proBNP levels.

$\mathrm{N}$-terminal-pro-B-type natriuretic peptide levels in either pleural fluid or serum showed a high diagnostic accuracy compared to traditional criteria. Thus measuring $\mathrm{N}$-terminal-pro-B-type natriuretic peptide is a valuable additional diagnostic tool for the detection or exclusion of cardiac origin of pleural effusions.

KEYWORDS: B-type natriuretic peptide, exudates and transudates, heart failure, pleural effusion

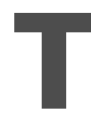

he differentiation of pleural effusions resulting from heart failure from those of other causes is usually made by clinical criteria supported by the finding of a transudative effusion according to the criteria of LIGHT [1] (table 1). However, as these criteria were developed to detect exudative pleural effusions with a high sensitivity for not overlooking underlying causes such as infections and malignancies, their ability to exclude transudative effusions is lower $[1,2]$. Some studies have found a considerable proportion of patients with pleural effusions due to heart failure being misclassified as exudative $[3,4]$, especially after having received diuretic therapy [5]. The finding of an exudative effusion usually requires an extensive diagnostic workup. Thus a diagnostic dilemma in patients with exudative pleural effusions and clinical heart failure might result, leading to an unnecessary exposure to invasive and expensive diagnostic procedures. Conversely, thoracentesis itself presents considerable risk of complications and is associated with discomfort to the patient. Thus a strategy of identifying pleural effusions due to heart failure and possibly avoiding unnecessary

For editorial comments see page 7. diagnostic thoracenteses and/or further diagnostic procedures would be an attractive and potentially beneficial approach.

B-type natriuretic peptide (BNP) is a vasoactive peptide predominantly secreted by the heart. Its precursor molecule, pro-BNP, is cleaved to give the inactive N-terminal-pro-BNP (NT-proBNP) and the biologically active BNP. The synthesis of these peptides is stimulated by increased tension or stretching of the cardiac ventricle wall. NTproBNP measured in serum is a sensitive marker of cardiac dysfunction and proven to be a useful tool in the diagnosis of acute and chronic systolic and diastolic left ventricular heart failure [6-9].

Since 2004, there has been some interest in the investigation of these peptides as markers for pleural effusions due to heart failure. To date, two studies have examined NT-proBNP $[10,11]$ and one BNP [12], with all uniformly suggesting a potential value of these peptides in predicting or ruling out heart failure as a cause of pleural effusions. There are, however, several limitations to these trials as they either measured the peptides only in pleural fluid [11] or plasma [12], were retrospective in design [11] or included only 28 selected patients to analyse [10].
AFFILIATIONS

*Dept of Pulmonology, Medical Clinic I, Carl Gustav Carus University Hospital, and

\# Dept of Cardiology, Medical Clinic II, Dresden University of Technology, Dresden, Germany.

CORRESPONDENCE M. Kolditz Dept of Pulmonology Medical Clinic I University Hospital Carl Gustav Carus Fetscherstr. 74 01307 Dresden Germany Fax: 493514585892 E-mail: martin.kolditz@uniklinikumdresden.de

Received:

September 282005 Accepted after revision: January 312006 


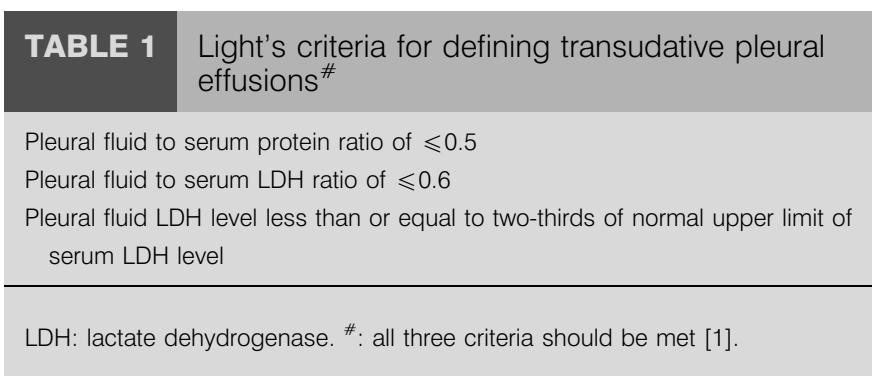

The aim of the present study was to prospectively evaluate the diagnostic accuracy of NT-proBNP levels, measured simultaneously in serum and pleural fluid, in identifying pleural effusions due to heart failure in all patients presenting for thoracentesis at the Dept of Pulmonology of Dresden University Hospital (Dresden, Germany) between April 2004 and May 2005.

\section{METHODS}

Pleural fluid and serum samples were collected prospectively from all patients presenting at the Dept of Pulmonology of Dresden University Hospital, a tertiary care referral centre for diagnostic or therapeutic thoracentesis, between April 2004 and May 2005. In patients presenting repeatedly for thoracentesis, only the first episode was included. Pleural fluid and serum samples were obtained, preferably simultaneously, but a maximum time difference of $\pm 8 \mathrm{~h}$ was accepted. Biochemical analysis, bacterial and fungal culture, acid-fast bacilli smear, PCR for Mycobacterium tuberculosis complex DNA, and cytological examinations (with flow cytometry if appropriate) were performed for all pleural fluid samples shortly after thoracentesis, whereas serum samples were sent for biochemical analysis. Measurement of NT-proBNP levels in serum and pleural effusion and all other biochemical analyses were carried out within $4 \mathrm{~h}$ after specimen collection.

Any further diagnostic work-up was left to the discretion of the attending physicians, but echocardiography was performed in all patients with suspected pleural effusion due to heart failure.

The study protocol was approved by the ethics committee of the medical faculty of Dresden University of Technology (Dresden, Germany), and informed consent was obtained from the participants.

Serum and pleural fluid total protein, lactate dehydrogenase $(\mathrm{LDH})$ and cholesterol levels were measured using test kits from Roche Diagnostics (Mannheim, Germany) and a Hitachi 917 analyser (Roche Diagnostics). The upper normal limit for serum LDH with the test kit used is $213 \mathrm{U} \cdot \mathrm{L}^{-1}$ in females and $225 \mathrm{U} \cdot \mathrm{L}^{-1}$ in males. NT-proBNP was measured by electrochemiluminescence immunoassay using the Elecsys 2010 (Roche Diagnostics) according to the manufacturer's protocol. According to the manufacturer, this test has an intra-assay coefficient of variation of $0.8-3 \%$ and a detection range of 5 $35,000 \mathrm{ng} \cdot \mathrm{L}^{-1}$. Higher values were obtained by diluting samples $1: 10$ or $1: 100$.

After the termination of the study, when the last included patient had been discharged from hospital, clinical diagnosis was established independently from the biochemical data by reviewing the hospital records of all patients.
The diagnosis of heart failure was based on findings of the typical clinical picture of decompensated heart failure, including history, physical examination, chest radiography and response to diuretic therapy, and confirmed by echocardiographical evidence of left ventricular systolic dysfunction (left ventricular ejection fraction of $\leqslant 40 \%$ ), severe valvular disease (grade II or III) or severe left ventricular diastolic dysfunction; all patients with pleural effusions due to heart failure had New York Heart Association Functional Class III or IV symptoms.

Malignant effusions were diagnosed when malignant cells were detected on cytological examination of pleural fluid or in lung biopsy specimens in the absence of other causes of pleural effusion.

Parapneumonic effusions or pleural empyemas were associated with the clinical and radiological diagnosis of acute pneumonia or the detection of pus or a positive bacterial culture in pleural fluid.

Other, rare, causes of exudative pleural effusions were defined by clear clinical pictures or established diagnostic criteria (post-cardiac injury syndrome, acute pleuritis, post-operative effusion, collagen disease, pleural tuberculosis, chylothorax).

Data from the biochemical analyses, including NT-proBNP levels, are presented as median (interquartile range). Medians were compared using the nonparametric Mann-Whitney Utest, and qualitative variables were compared using Fisher's exact test. The correlation between pleural fluid and serum NT-proBNP concentration was tested using Spearman's coefficient of rank correlation. Sensitivity, specificity, positive predictive value, negative predictive value and accuracy were calculated according to standard formulae. Exact binomial $95 \%$ confidence intervals (CI) were calculated for all operational characteristics. Receiver operating characteristic (ROC) curve analysis was used to determine the discriminative properties of various cut-off levels of NT-proBNP. A p-value of $<0.05$ (two-sided) was considered significant. The regression equation was calculated as described previously according to the PASSING and BABLOK [13] method.

\section{RESULTS}

Pleural fluid and serum samples were collected from 101 consecutive patients presenting for thoracentesis at the Dept of Pulmonology between April 2004 and May 2005. Eight (8\%) patients, for whom a definite diagnosis could not be established from the hospital records, were excluded from the analysis. The remaining 93 patients were divided as follows, according to the aforementioned criteria, amongst the following diagnoses: $25(27 \%)$ with effusions due to heart failure, $40(43 \%)$ with malignant effusions, $15(16 \%)$ with parapneumonic effusions or pleural empyema, and $13(14 \%)$ with exudative pleural effusions due to other causes (four acute pleuritis, three post-cardiac injury syndrome, three after local surgery, one collagen disease, one pleural tuberculosis and one chylothorax).

Of the patients diagnosed with pleural effusion due to heart failure, $18(72 \%)$ suffered from systolic heart failure with a median (interquartile range) left ventricular ejection fraction of $30 \%(25-40 \%)$, four $(16 \%)$ patients had acute decompensated left ventricular valvular defects of grade II and III and three $(12 \%)$ patients presented with lung congestion due to severe 
left ventricular diastolic dysfunction associated with pulmonary venous hypertension, established by heart catheter examination.

As expected, patients with cardiac transudates were of greater age, more often had a history of chronic heart failure, myocardial infarction, renal failure or diabetes mellitus, more often presented with bilateral effusions and had significantly lower pleural fluid levels and pleural fluid to serum ratios of protein, LDH and cholesterol (table 2). However, patients with exudative effusions frequently suffered from comorbid illnesses, with $27(40 \%)$ of them having a history of chronic, nondecompensated heart failure.

Levels of NT-proBNP measured in pleural fluid and in serum correlated closely (Spearman's coefficient of rank correlation 0.96; $\mathrm{p}<0.001$; fig. 1).

Median (interquartile range) levels of NT-proBNP in pleural fluid or serum among patients with pleural effusion due to heart failure were significantly higher $(10,427 \quad(7,366-$ $21,844) \mathrm{ng} \cdot \mathrm{L}^{-1}$ in pleural fluid; $10,791(6,588-20,263) \mathrm{ng} \cdot \mathrm{L}^{-1}$ in serum) than in patients with noncardiac causes (947 (372$1,937) \mathrm{ng} \cdot \mathrm{L}^{-1}$ in pleural fluid; $989(296-1,691) \mathrm{ng} \cdot \mathrm{L}^{-1}$ in serum; all $\mathrm{p}<0.0001$; fig. 2, table 2).

Measurement of NT-proBNP levels in pleural fluid and serum displayed high diagnostic accuracy, as shown by ROC curve analysis (area under the curve 0.98 for both; fig. 3). Linear discriminant analysis after logarithmic transformation of NTproBNP levels revealed nearly identical cross-validated

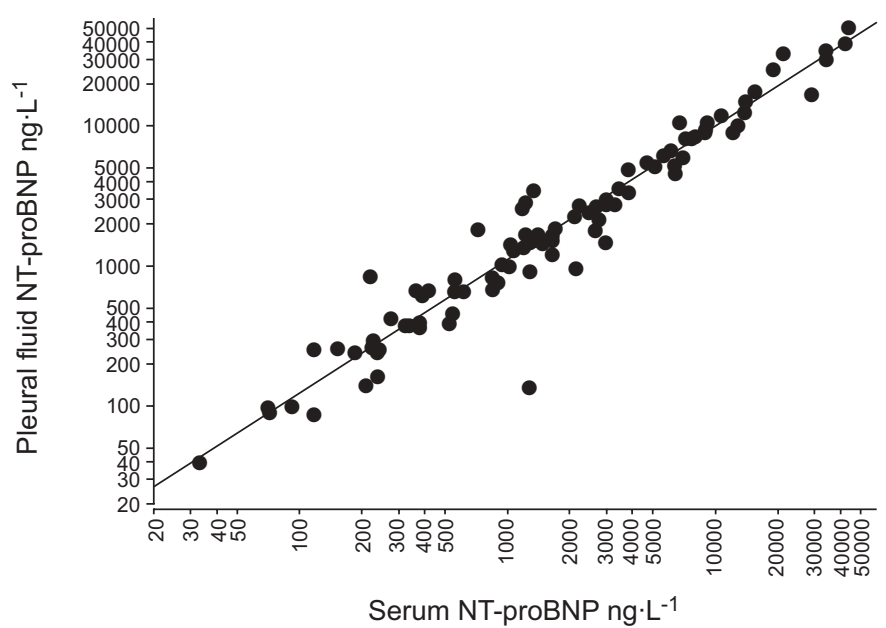

FIGURE 1. Correlation of serum and pleural effusion levels of N-terminalpro-B-type natriuretic peptide (NT-proBNP). Spearman's coefficient of rank correlation is 0.963 (95\% confidence interval (Cl) 0.944-0.975; $p<0.001$ ). Regression equation according to PASSING and BABLOK [13] method: serum NT-proBNP $=-12.967+0.959 \times$ pleural fluid NT-proBNP; intercept $A=-12.967(95 \% \mathrm{Cl}-59.000-$ 12.414); slope $B=0.959$ (95\% $\mathrm{Cl} 0.908-1.000)$. The scale is a logarithmic scale.

discriminative properties for measurement in pleural fluid and serum. As determined by ROC curve analysis, an NTproBNP cut-off value of $4,000 \mathrm{ng} \cdot \mathrm{L}^{-1}$ in pleural fluid and serum had a sensitivity of $92 \%$ (95\% CI 74-99\%) and $88 \%$ (95\% CI 69$97 \%$ ) and a specificity of $93 \%$ (95\% CI $84-98 \%$; both the same),

\section{TABLE 2 Characteristics of study participants}

\begin{tabular}{|c|c|c|c|}
\hline Subjects $\mathbf{n}$ & 25 & 68 & \\
\hline Age yrs & $72(64-83)$ & $65(56-76)$ & 0.027 \\
\hline Male & $18(72)$ & $36(53)$ & 0.15 \\
\hline Bilateral effusions & $15(60)$ & $22(32)$ & 0.019 \\
\hline History of renal failure & $11(44)$ & $11(16)$ & 0.011 \\
\hline History of diabetes mellitus & $12(48)$ & $13(19)$ & 0.008 \\
\hline \multicolumn{4}{|l|}{ Biochemical data } \\
\hline Pleural fluid protein $\mathrm{g} \cdot \mathrm{L}^{-1}$ & $20.4(16.7-24.7)$ & $35.6(27.2-41.4)$ & $<0.001$ \\
\hline Pleural fluid/serum protein ratio & $0.32(0.27-0.46)$ & $0.56(0.45-0.63)$ & $<0.001$ \\
\hline Pleural fluid NT-proBNP $n g \cdot L^{-1}$ & $10427(7366-21844)$ & $947(372-1937)$ & $<0.001$ \\
\hline Serum NT-proBNP $n g \cdot L^{-1}$ & 10791 (6588-20263) & $989(296-1691)$ & $<0.001$ \\
\hline \multicolumn{4}{|l|}{ Light's criteria } \\
\hline Transudative effusion & $16(64)$ & $5(7)$ & $<0.001$ \\
\hline One exudative criterion & $5(20)$ & $15(22)$ & 1.0 \\
\hline Two exudative criteria & $2(8)$ & $16(24)$ & 0.139 \\
\hline Three exudative criteria & $2(8)$ & $32(47)$ & $<0.001$ \\
\hline
\end{tabular}




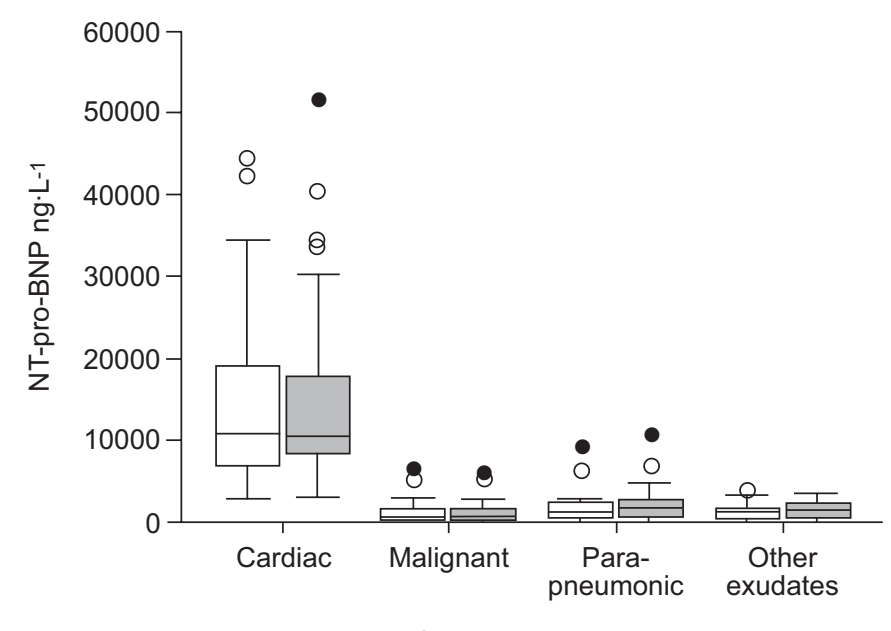

Clinical diagnosis

FIGURE 2. Box-plots showing N-terminal-pro-B-type natriuretic peptide (NTproBNP) levels in serum $(\square)$ and pleural effusion $(\square)$ by clinical diagnosis. Boxes represent median and interquartile range $(\bigcirc$ : outliers;

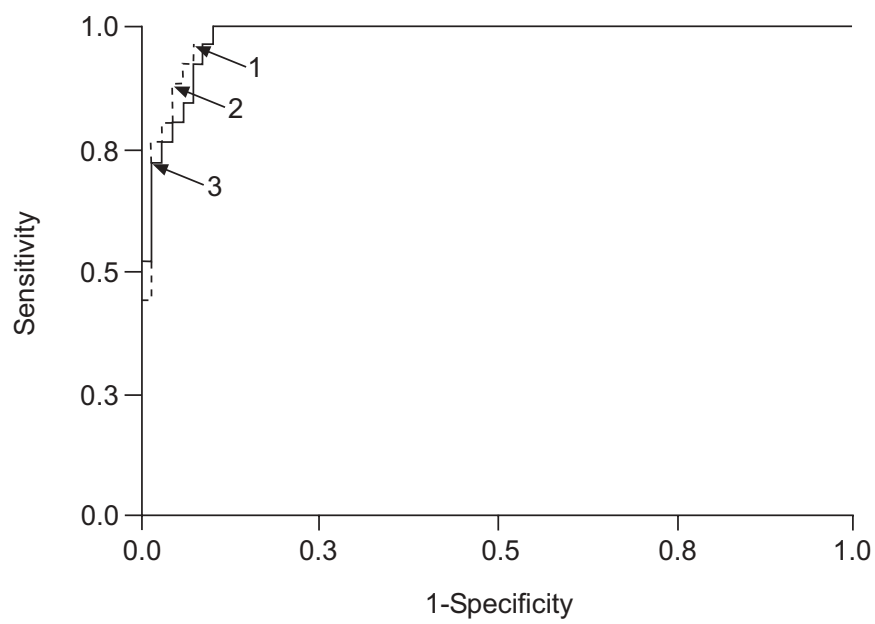

FIGURE 3. Receiver operating characteristic curve of N-terminal-pro-B-type natriuretic peptide (NT-proBNP) levels $\left(1: 3,000 \mathrm{ng} \cdot \mathrm{L}^{-1} ; 2: 4,000 \mathrm{ng} \cdot \mathrm{L}^{-1} ; 3\right.$ : $\left.7,000 \mathrm{ng} \cdot \mathrm{L}^{-1}\right)$ in serum $(-)$ and pleural effusion (-----) for differentiating between cardiac and noncardiac pleural effusions (area under the curve 0.98 for both serum and pleural effusion (95\% confidence interval 0.96-1.00)).

each with an overall diagnostic accuracy of 92\% (95\% CI 8597\%) and 91\% (95\% CI 84-96\%), respectively (table 3).

In the present patient group, where transudative effusions were caused by heart failure alone, it was possible to calculate the comparative diagnostic properties of Light's criteria in detecting cardiac effusions in a post-hoc analysis: the finding of a transudate was associated with a low sensitivity of $64 \%(95 \%$ CI $43-82 \%)$. As expected, their specificity in the exclusion of exudative effusions was high at 93\% (95\% CI 84-98\%), leading to an overall diagnostic accuracy of $85 \%$ (95\% CI 76-92\%). Nine of the $25(36 \%)$ patients with pleural effusion due to heart failure were falsely classified as exudative by Light's criteria. All of them exhibited NT-proBNP levels of $>4,000 \mathrm{ng} \cdot \mathrm{L}^{-1}$. The characteristics of these nine patients are shown in table 4 ; all

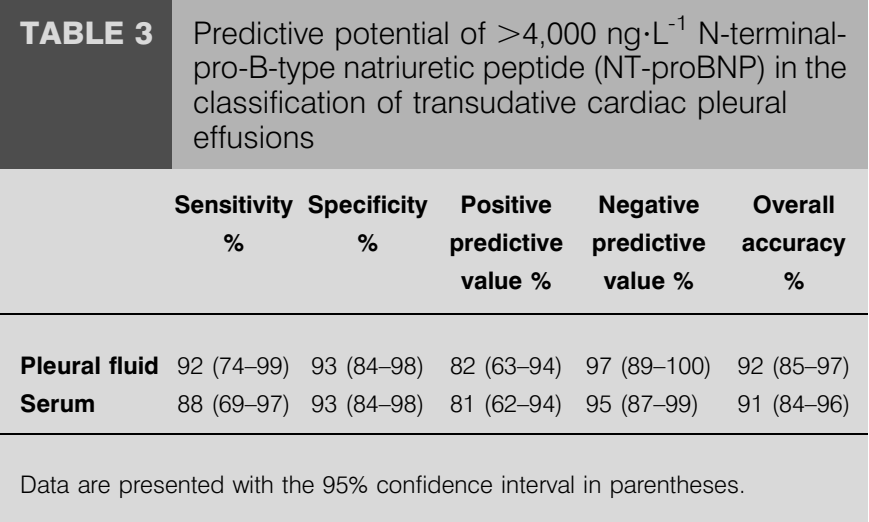

except one received diuretic therapy. Conversely, all five patients with exudates falsely labelled as transudates by Light's criteria showed NT-proBNP levels of $<4000 \mathrm{ng} \cdot \mathrm{L}^{-1}$, three of them having confirmed malignant effusions.

\section{DISCUSSION}

The serum NT-proBNP level is an established marker for the assessment of cardiac function, and has successfully been used as a tool for the diagnosis and management of acute and chronic heart failure, including systolic and diastolic left ventricular dysfunction and valvular disease [6-9, 14]. Especially in the emergency care setting, NT-proBNP levels are a valuable addition to clinical judgement for the identification or exclusion of acute heart failure as a cause of dyspnoea [6]. Measurement of BNP and NT-proBNP in serum were shown to perform equally well in predicting acute heart failure [15].

In patients presenting with pleural effusion, the current findings demonstrate that levels of NT-proBNP in serum and pleural fluid are significantly elevated, $\sim 10$-fold, in patients in whom acute decompensated left heart failure could be identified as the cause of the effusion compared to patients with noncardiac effusions. Moreover, elevated NT-proBNP levels displayed a high sensitivity and specificity in detecting cardiac transudates in this patient group over a relatively large range of cut-off values (fig. 3).

Compared with the finding of a transudate using the established Light's criteria in the present patient group, in whom transudative pleural effusions were only attributed to heart failure, it was found that NT-proBNP levels in serum and pleural fluid discriminate more accurately between cardiac versus noncardiac effusions. In accordance with the literature, Light's criteria exhibited high ability in the exclusion of exudates, but the finding of a transudate showed a low sensitivity of $64 \%$ in the identification of cardiac effusions. In patients misclassified by Light's criteria in either way, NTproBNP levels were always able to correctly detect or exclude a cardiac origin of the effusion. Thus Light's criteria remain the diagnostic standard for the exclusion of exudative effusions, but measurement of NT-proBNP levels proved to be a valuable supplementary diagnostic tool, which, according to the present data, strongly suggests a cardiac origin of the effusion at levels $>4,000 \mathrm{ng} \cdot \mathrm{L}^{-1}$, and, conversely, makes cardiac effusions very unlikely at levels of $<4,000 \mathrm{ng} \cdot \mathrm{L}^{-1}$ (fig. 3). These data are in general agreement with the other studies examining this issue. 


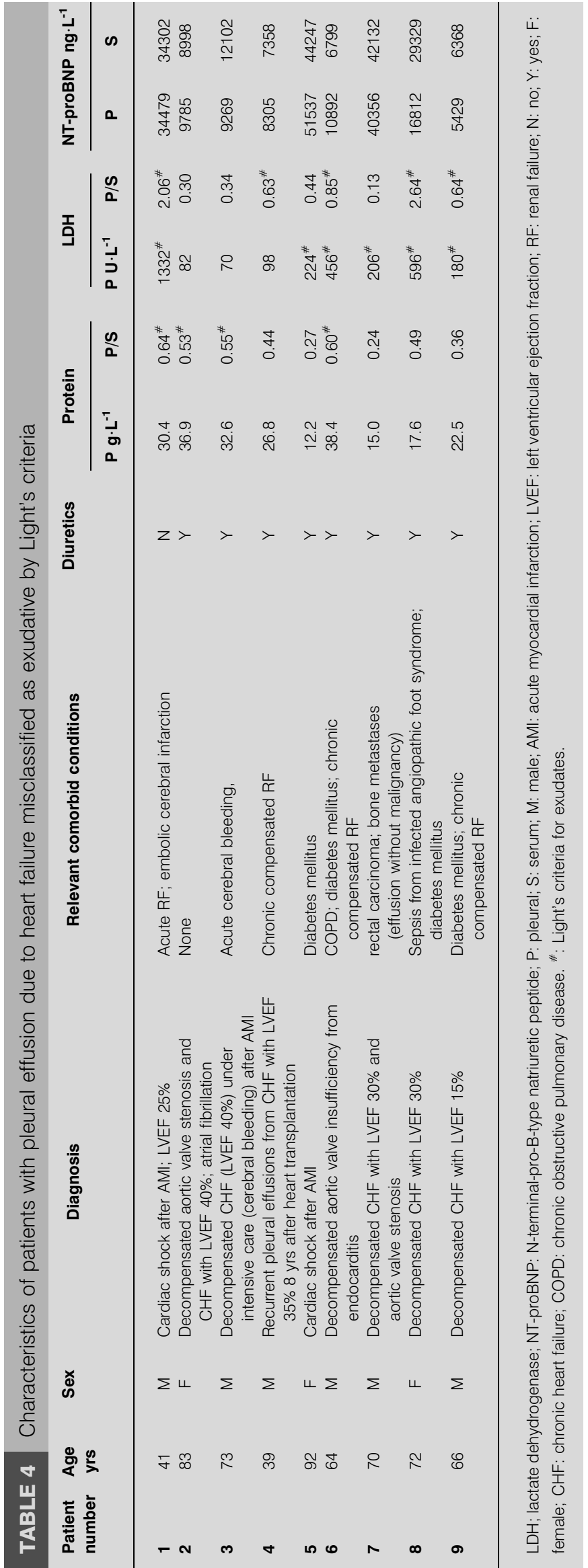

One recently published study by GEGENHUBER et al. [12] examined plasma levels of BNP in 64 prospectively enrolled consecutive patients, 31 with pleural effusions due to heart failure, two with transudative effusions of different origin and 24 with exudative effusions. They reported a diagnostic accuracy for plasma BNP levels of $93 \%$ in the identification of cardiac effusions, comparable to the data found in the present study with NT-proBNP.

TOMCSANYI et al. [10] prospectively compared NT-proBNP levels in pleural fluid and serum in 14 patients with pleural effusion due to congestive heart failure and 14 patients with pleural exudates of various cause. In this small study, the authors found significantly higher levels of NT-proBNP in either serum or pleural fluid from patients with cardiac effusions (median 6,295 versus $277 \mathrm{ng} \cdot \mathrm{L}^{-1}$ in pleural fluid; 5,713 versus $236 \mathrm{ng} \cdot \mathrm{L}^{-1}$ in serum) and suggested a diagnostic cut-off point for detecting cardiac transudates of $599-1,457 \mathrm{ng} \cdot \mathrm{L}^{-1}$. However, patients with exudative effusions and coexisting chronic heart failure were excluded from their analysis.

PorCEL et al. [11] retrospectively examined NT-proBNP levels in the pleural fluid of a cohort of 117 patients after thoracentesis, randomly selected from a larger database, of whom $44(38 \%)$ were diagnosed as having pleural effusion due to acute heart failure. Consistent with the present data, the authors detected significantly higher median levels of NTproBNP in cardiac effusions $\left(6,931 \mathrm{ng} \cdot \mathrm{L}^{-1}\right)$ compared to exudative effusions $\left(292 \mathrm{ng} \cdot \mathrm{L}^{-1}\right)$. From their data, they calculated a diagnostic cut-off point of $1,500 \mathrm{ng} \cdot \mathrm{L}^{-1}$ for detecting cardiac transudates with a sensitivity of $91 \%$ and a specificity of $93 \%$. NTproBNP levels in serum were not measured in their study.

As in the present study, both authors measured NT-proBNP levels using a commercial electrochemiluminescence immunoassay and an Elecsys 2010 analyser (Roche Diagnostics).

Although the NT-proBNP levels of the present patients with pleural effusions due to heart failure are comparable to those determined by the former two groups, approximately threefold higher median NT-proBNP levels were detected in the present patients with exudative effusions (table 2), resulting in a suggested cut-off value that is approximately three-fold higher. Unlike the other two studies, the present prospective data present the results from nonselected consecutive patients with pleural effusion. This led to the inclusion of a considerable proportion $(40 \%)$ of patients with noncardiac exudative effusions suffering from coexisting mild-to-moderate chronic but not decompensated heart failure, including chronic right and/or left ventricular dysfunction, chronic cardiac valve disease and/or chronic atrial fibrillation (table 2), conditions known to be associated with elevated NT-proBNP levels. Moreover, of the present patients with exudative effusions, $19 \%$ had a history of diabetes mellitus and $16 \%$ of impaired renal function, diseases that can also cause elevated NTproBNP-levels [16]. The discrepancy in NT-proBNP levels might be explained by the high frequency of these comorbid conditions in the present nonselected patient group, frequently suffering from thoracic malignancies and pleural empyema, which mainly occur in elderly patients with multiple comorbid conditions. Thus they possibly represent a more realistic group of control subjects. This suggestion is supported by the finding 
of a study evaluating NT-proBNP concentrations in serum as a diagnostic tool for the detection of reduced left ventricular systolic function in a cohort of 2,193 consecutive hospital inpatients [17]. The authors divided the patients into three groups and found median NT-proBNP levels of $7,273 \mathrm{ng} \cdot \mathrm{L}^{-1}$, $2,368 \mathrm{ng} \cdot \mathrm{L}^{-1}$ and $685 \mathrm{ng} \cdot \mathrm{L}^{-1}$ in patients with an LVEF of $\leqslant 40 \%$, $>40 \%$ and $>50 \%$, respectively. Based on this data from consecutive hospitalised patients, the median serum NTproBNP level of $989 \mathrm{ng} \cdot \mathrm{L}^{-1}$ seen in the present patient group with noncardiac pleural effusions and a high frequency of comorbid conditions, including nondecompensated chronic heart failure, lies well within the expected range. Thus, the present authors' suggested a higher cut-off value of $4,000 \mathrm{ng} \cdot \mathrm{L}^{-1}$ might be more appropriate in accurately predicting a cardiac origin of pleural effusions while minimising of the risk of overlooking additional underlying diseases. Conversely, the possibility that the present results and the corresponding cut-off values have also been biased due to the limitations of the study, discussed below, cannot be excluded. Thus further prospective trials on large nonselected patient groups are needed in order to confirm these issues.

The other interesting finding of the present study is the close correlation between NT-proBNP levels in pleural fluid and serum, leading to equal diagnostic efficacy in the identification of cardiac effusions. This confirms the data of TOMCSANYI et al. [10], who found a comparably high correlation. To date, the origin of NT-proBNP in pleural fluid is unclear, although it has been suggested that it derives from serum NT-proBNP, and might diffuse easily into the pleural space due to its small molecular size [18]. Thus there seems to be no additional value in measuring NT-proBNP in pleural fluid.

There are several limitations to the present study. First, the examination of a relatively small prospective group of 93 consecutive patients means that the present results need to be confirmed in larger studies.

Secondly, since the study was designed as a pilot study to prospectively examine the diagnostic discriminative properties of NT-proBNP levels between cardiac and noncardiac pleural effusions and to suggest cut-off values for further studies, the small subset of eight patients in whom a definite diagnosis could not be established from the hospital records had to be excluded from the analysis, although this might be a subgroup of potential interest in studying the predictive properties of NT-proBNP level.

Thirdly, it was not possible to include any transudative pleural effusions of noncardiac origin, such as effusions associated with hepatic or renal disease. Examining the diagnostic utility of NT-proBNP level in this patient group would be of particular interest, since Light's criteria are not able to discriminate between them. Moreover, the preliminary data from PORCEL et al. [11] suggest a potential value of NT-proBNP level for this indication.

Fourthly, as the diagnosis of pleural effusion due to heart failure is usually based on clinical criteria supported by the finding of a transudate, it cannot be concluded, from the present data, whether or not NT-proBNP measurement would be of any additional diagnostic value compared to the clinical impression of the treating physician; this issue remains subject to further studies [18].

Fifthly, there were several factors related to the study design potentially biasing the present results. Echocardiography was performed in all patients with suspected pleural effusion due to heart failure; in all other patients this was left to the discretion of the attending physician. Thus, in a minority of 25 of the 68 patients diagnosed as having noncardiac effusions, who were all without any clinical signs suggestive of heart failure, echocardiography was not performed and underlying heart disease, although less probable, cannot be entirely excluded. As a small proportion of patients with pleural effusion due to heart failure or malignancy referred for therapeutic rather than diagnostic thoracentesis were included, this might have biased the disease prevalence of heart failure in the present patient group and thus the diagnostic properties calculated for NT-proBNP level. Finally, although the studyrelevant diagnosis was established independently from the biochemical data, the attending physicians treating the patients were not blinded to the NT-proBNP results. The possibility cannot be excluded that this diagnostic information might have influenced further diagnostic approaches and thus, again, potentially biased the study results.

In conclusion, NT-proBNP levels in either pleural fluid or serum showed high diagnostic accuracy in identifying cardiac transudates. Thus they might be valuable additional diagnostic tools for the detection or exclusion of a cardiac origin of pleural effusions. From the present data, it can be suggested that cutoff levels in nonselected patient groups might be higher than those previously reported $[10,11]$ to avoid overlooking underlying causes other than heart failure in the often multimorbid patients presenting with pleural effusions. However, larger prospective studies are needed to confirm the cut-off points in nonselected patient groups and to examine the discriminatory properties of NT-proBNP level in differentiating transudates of noncardiac origin. It would also be interesting to compare the diagnostic properties of NT-proBNP level with the clinical suspicion of the treating physician in addition to the finding of Light's criteria in the identification of cardiac effusions.

As N-terminal-pro-B-type natriuretic peptide levels in serum and pleural fluid correlate closely, and measurement of $\mathrm{N}$ terminal-pro-B-type natriuretic peptide in serum showed equally good diagnostic properties, examination in serum alone might be a promising diagnostic tool for the suggestion of pleural effusion due to heart failure with an acceptable positive predictive value, leading to the possibility of potentially postponing diagnostic thoracentesis in this situation until a trial of diuretic therapy has been performed. However, this strategy should be addressed in further prospective trials.

\section{REFERENCES}

1 Light RW. Pleural effusion. N Engl J Med 2002; 346: 1971-1977.

2 Romero S, Candela A, Martin C, Hernandez L, Trigo C, Gil J. Evaluation of different criteria for the separation of pleural transudates from exudates. Chest 1993; 104: 399-404. 
3 Gotsman I, Fridlender Z, Meirowitz A, Dratva D, Muszkat M. The evaluation of pleural effusions in patients with heart failure. Am J Med 2001; 111: 375-378.

4 Heffner JE, Sahn SA, Brown LK. Multilevel likelihood ratios for identifying exudative pleural effusions. Chest 2002; 121: 1916-1920.

5 Chakko SC, Caldwell SH, Sforza PP. Treatment of congestive heart failure. Its effect on pleural fluid chemistry. Chest 1989; 95: 798-802.

6 Januzzi JL Jr, Camargo CA, Anwaruddin S, et al. The Nterminal Pro-BNP Investigation of Dyspnea in the Emergency department (PRIDE) study. Am J Cardiol 2005; 95: 948-954.

7 Hobbs FD, Davis RC, Roalfe AK, Hare R, Davies MK, Kenkre JE. Reliability of N-terminal pro-brain natriuretic peptide assay in diagnosis of heart failure: cohort study in representative and high risk community populations. BMJ 2002; 324: 1498-1502.

8 McDonagh TA, Holmer S, Raymond I, Luchner A, Hildebrandt P, Dargie HJ. NT-proBNP and the diagnosis of heart failure: a pooled analysis of three European epidemiological studies. Eur J Heart Fail 2004; 6: 269-273.

9 Tschope C, Kasner M, Westermann D, Gaub R, Poller WC, Schultheiss HP. The role of NT-proBNP in the diagnosis of isolated diastolic dysfunction: correlation with echocardiographic and invasive measurements. Eur Heart J 2005; 26: 2277-2284.

10 Tomcsanyi J, Nagy E, Somloi M, et al. NT-brain natriuretic peptide levels in pleural fluid distinguish between pleural transudates and exudates. Eur J Heart Fail 2004; 6: 753-756.

11 Porcel JM, Vives M, Cao G, Esquerda A, Rubio M, Rivas MC. Measurement of pro-brain natriuretic peptide in pleural fluid for the diagnosis of pleural effusions due to heart failure. Am J Med 2004; 116: 417-420.

12 Gegenhuber A, Mueller T, Dieplinger B, Lenz K, Poelz W, Haltmayers M. Plasma B-type natriuretic peptide in patients with pleural effusions: preliminary observations. Chest 2005; 128: 1003-1009.

13 Passing $\mathrm{H}$, Bablok $\mathrm{W}$. A new biometrical procedure for testing the equality of measurements from two different analytical methods. Application of linear regression procedures for method comparison studies in clinical chemistry, part I. J Clin Chem Clin Biochem 1983; 21: 709-720.

14 Weber M, Arnold R, Rau M, et al. Relation of N-terminal pro B-type natriuretic peptide to progression of aortic valve disease. Eur Heart J 2005; 26: 1023-1030.

15 Mueller T, Gegenhuber A, Poelz W, Haltmayer M. Diagnostic accuracy of $\mathrm{B}$ type natriuretic peptide and amino terminal proBNP in the emergency diagnosis of heart failure. Heart 2005; 91: 606-612.

16 Raymond I, Groenning BA, Hildebrandt PR, et al. The influence of age, sex and other variables on the plasma level of $\mathrm{N}$-terminal pro brain natriuretic peptide in a large sample of the general population. Heart 2003; 89: 745-751.

17 Bay M, Kirk V, Parner J, et al. NT-proBNP: a new diagnostic screening tool to differentiate between patients with normal and reduced left ventricular systolic function. Heart 2003; 89: 150-154.

18 Zemans RL, Chatterjee K, Matthay A. Diagnostic utility of B-type natriuretic peptide in patients with acute dyspnea or pleural effusions. Am J Med 2004; 116: 424-426. 